\title{
Subset prediction in multiple-response probability learning
}

\author{
RICHARD B. MILLWARD, Brown \\ University, Providence, R.I. 02912
}

Two groups of $S s$, varying in reinforcement probabilities, were run in a four-response probability-learning task. After 320 trials, they were required to respond with less than the four responses. The constant ratio, additive and equal probability rules were tested as possible ways of describing how $S_{s}$ redistribute the response probabilities of prohibited responses. The additive rule was best overall but still a very poor fit.

The most prevalent interpretation of probability learning (PL) has been in terms of linear-operator models. This interpretation assumes that each of the $r$ response alternatives has a strength or probability associated with it. The question posed here is what happens to the response probability associated with a response when that response is eliminated from the response set or prohibited from occurring. Three rules for determining the new response probabilities of the remaining responses are considered. Let the original set of four responses, $S$, be $\{i, j, m, n\}$. Let $\mathrm{U}=\left\{\mathrm{i}, \mathrm{j}, \mathrm{m}^{;}\right.$, be the subset when $\mathrm{n}$ is eliminated and $T=\{i, j\}$ be the subset when both $m$ and $n$ are eliminated. Let $x$ be a member of the subset under consideration. The three rules are:

\section{(1) Constant-ratio:}

$P(x \mid U)=P(x \mid S) /[1-P(n \mid S)]$, $P(x \mid T)=P(x \mid S) /[1-P(n \mid S)-P(m \mid S)]$.

(2) Additive:

$P(x \mid U)=P(x \mid S)+1 / 3 P(n \mid S)$, $P(x \mid T)=P(x \mid S)+1 / 2[P(n \mid S)+P(m \mid S)]$.

(3) Equal-probability:

$P(x \mid U)=1 / 3$,

$P(x \mid T)=1 / 2$.

It can be shown that the additive rule leads to values that lie between the values for the constant-ratio and equal-probabilities rule. We show this for the case, $P(x \mid U)_{1} \geqslant 1 / 3$, where the subscript denotes the rule. We wish to show that $\mathrm{P}(\mathrm{x} \mid \mathrm{U})_{1} \geqslant \mathrm{P}(\mathrm{x} \mid \mathrm{U})_{2}$ $\geqslant 1 / 3$. Note, $P(x \mid U)_{1} \geqslant 1 / 3$ implies $3 P(x \mid S) \geqslant 1-P(n \mid S)$. Assume that $P(x \mid U)_{2} \geqslant P(x \mid U)_{1} \geqslant 1 / 3$, or by the rules, $P(x \mid S)+1 / 3 P(n \mid S)>$
$P(x \mid S) /[1-P(n \mid S)]$, i.e., the contradiction of what we wish to show. Then, by simple algebra, the assumption leads to $3 P(x \mid S) \leqslant 1-P(n \mid S)$, which contradicts the implication noted above. If we assume that $P(x \mid U)_{1} \leqslant 1 / 3$, a similar argument shows that $P(x \mid U)_{2}$ lies between $P(x \mid U)_{1}$ and $1 / 3$. The same proof can be carried out for the case where two responses are eliminated. The importance of the order of the predictions of these rules will be discussed below.

\section{METHOD}

Forty-five students from an introductory psychology class at Brown University served for $2 \mathrm{~h}$ each in order to meet a class requirement and to earn $\$ 1.00$. The stimuli consisted of four nonsense syllables (NS) placed randomly in a matrix of 3 by 3 cells. The four NSs were LAJ, GIW, MEF, and YOP. Of the possible $9 \cdot 8 \cdot 7 \cdot 6$ permutations of the four NSs in the nine cells, 160 were randomly selected and made into slides. There are six proper subsets of two NSs each and four subsets of three NSs each. Eight different slides of each of the 10 possible subsets were made.

Two groups were run. Group 1 had a reinforcement schedule for the four NSs of $.4, .3, .2$, and .1 . Group 2 had a schedule with $.6, .2, .1$, and .1 . Ss were presented with 320 reinforced trials (twice through the 160 slides). The next 320 trials consisted of alternations between reinforced trials (slides with four NSs) and test trials, with slides containing either a subset of three NSs or a subset of two NSs. There were 160 test trials of the 10 subsets, or 16 test trials for each subset. Each of the eight subset slides for a given subset was presented twice, once during the first 80 test trials and once during the second 80 test trials. For each group, there were two subgroups that differed in the randomized order of the reinforcements. The sequence of slides remained the same. The four subgroups had 10,12,10, and 13 Ss, respectively. All Ss in each subgroup were run at one time. The stimuli were shown with a Kodak Carousel projector on a screen in front of a classroom. Ss recorded their responses in a booklet in such a way that the past responses were covered after every 10 responses.

\section{RESULTS}

Table 1 presents the asymptotic proportion of LAJ, GIW, MEF, and YOP responses over the last 160 reinforced trials. Group 1 Ss attained response proportions very close to the reinforced values. This result confirms predictions from simple linear operator-type models. However, Group 2 Ss showed a substantially higher proportion of LAJ responses that were reinforced $60 \%$ of the time. The .20 and .10 responses were accordingly too low. Such overshooting has been found previously in multiple-response PL, although only a few studies have been run with multiple responses. The amount of overshooting seems to be related to some aspect of the variance of the reinforcing probabilities. Further work is needed to clarify the exact relationship.

Table 2 presents the observed proportions for the four responses and for the responses on subset test trials for the last 160 trials. The frequencies used in determining these proportions are listed. In Table 2, the predictions of the constant-ratio and additive rules are also presented. The predictions of the equal-probability rule are simply $1 / 3$ for three-response subsets and $1 / 2$ for two-response subsets. It yielded an extremely poor fit in all cases. The chi-square values are determined in the usual way and give a gross measure of goodness of fit of the two models. In almost all cases, the additive rule provides the best fit, and it gives the overall smallest chi square. Since there are $2 \mathrm{dfs}$ for the three-response subsets and $1 \mathrm{df}$ for the two-response subsets, there are $14 \mathrm{dfs}$ for each of the summed chi squares. These chi squares are legitimate only if we assume that each test trial is independent. We have combined across Ss and across repeated measures for the same $S$, thus violating the independence assumption at least at a data-collection level.

\section{DISCUSSION}

These results must be accepted with some reservation due to the fact that the

. Table 1

Asymptotic Response Proportions for the Last 160 Trials

\begin{tabular}{|c|c|c|c|c|c|c|c|c|}
\hline \multirow[b]{2}{*}{$\begin{array}{c}\text { Reinforcement } \\
\text { Value }\end{array}$} & \multicolumn{4}{|c|}{$\begin{array}{c}\text { Group } 1 \\
\text { Responses }\end{array}$} & \multicolumn{4}{|c|}{$\begin{array}{c}\text { Group } 2 \\
\text { Responses }\end{array}$} \\
\hline & $\begin{array}{c}\text { LAJ } \\
.4\end{array}$ & $\begin{array}{c}\text { GIW } \\
.3\end{array}$ & $\begin{array}{l}\text { MEF } \\
.2\end{array}$ & $\begin{array}{c}\text { YOP } \\
.1\end{array}$ & $\begin{array}{c}\mathrm{LAJ} \\
.6\end{array}$ & $\begin{array}{c}\text { GIW } \\
.2\end{array}$ & $\begin{array}{c}\text { MEF } \\
. I\end{array}$ & $\begin{array}{c}\text { YOP } \\
.1\end{array}$ \\
\hline Block 9 & .38 & .31 & .18 & .13 & .73 & .14 & .07 & .06 \\
\hline Block 10 & .41 & .32 & .15 & .12 & .73 & .15 & .06 & .07 \\
\hline Block 11 & .41 & .31 & .18 & .10 & .76 & .11 & .06 & .07 \\
\hline Block 12 & .40 & .37 & .13 & .11 & .75 & .14 & .06 & .06 \\
\hline
\end{tabular}


Table 2

Predictions and Goodness of Fit of Two Rules for Subset Proportions

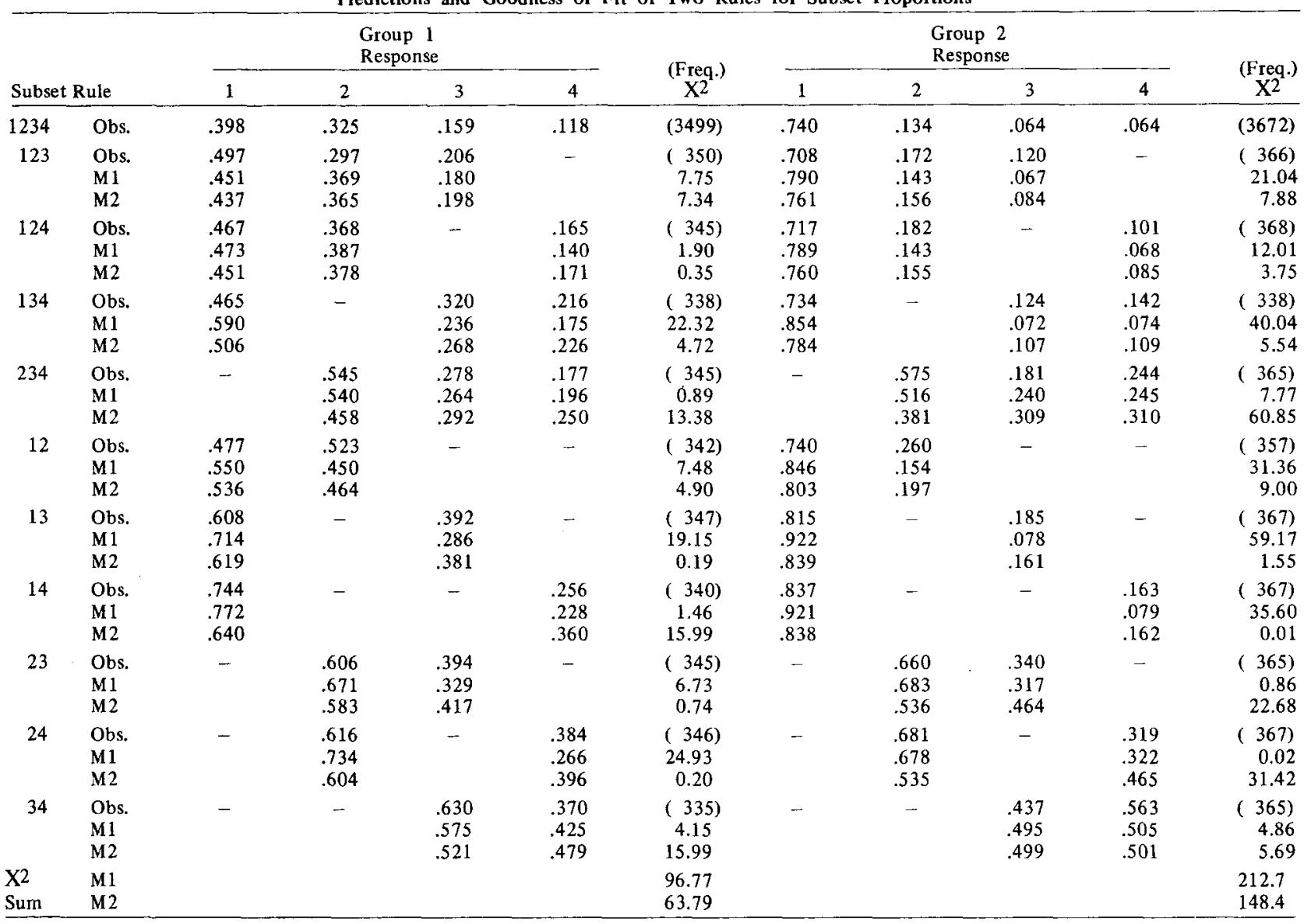

Obs. stands for observed proportions, $M 1$ for Rule 1 , and $M 2$ for Rule 2 proportions. Under the Freq. column, for the observed rows, the total frequency is listed while for Rule 1 and Rule 2 rows the $X^{2}$ value is listed.

additive rule predicts results between the ratio and the equal-probability rules. Thus, if some Ss follow the ratio rule and others the equal-probability rule, or if on some trials, a given $S$ guesses but follows the ratio rule on other trials, the present results might well occur. These results could be an artifact of averaging. A more refined experiment is needed in which individual Ss with sufficiently large frequencies are used to test the three rules.
A second problem lies much deeper. It is possible that the assumption of a single quantity, response probability, divided among alternative responses is inadequate. It could be that the changed stimulus conditions under the subset test trials make Ss behave idiosyncratically. A more complex decision process may be required. Thus, the particular transfer rules required by a situation involving subset predictions could be critical in evaluating the type of learning models appropriate for the learning in general. Finally, although the additive rule is a better rule for our results, we cannot consider it a very good predictor in an absolute sense.

\section{NOTE}

1. This research was supported in part by a grant from the National Institute of Mental Health, No. 11255. The author wishes to thank Beverly Alpert, who assisted in collecting the data for this experiment. 